\title{
On Capacity Scaling Law of Cognitive Radio Ad Hoc Networks
}

\author{
(Invited Paper) \\ Yi Shi Canming Jiang Y. Thomas Hou \\ The Bradley Department of Electrical and Computer Engineering \\ Virginia Polytechnic Institute and State University \\ Blacksburg, VA, USA
}

\author{
Sastry Kompella \\ Information Technology Division \\ U.S. Naval Research Laboratory \\ Washington, DC, USA
}

\begin{abstract}
Cognitive radio is envisioned to be an enabling radio technology for future wireless networks. In this paper, we study the capacity scaling laws for cognitive radio ad hoc networks (CRNs), i.e., how each individual node's capacity scales as the number of nodes in the network increases. This effort is critical to the fundamental understanding of the scalability of such network. However, due to the heterogeneity in available frequency bands at each node, the asymptotic capacity is much more difficult to develop than prior efforts for other types of wireless networks. To overcome this difficulty, we introduce two auxiliary networks $\zeta$ and $\alpha$ to analyze the capacity upper bound and lower bound. We derive the capacity results under both the protocol model and the physical model. Further, we show that the results developed by Gupta and Kumar for the simple single-channel single-radio (SC-SR) networks are special cases under the results for CRNs.
\end{abstract}

Index Terms-Cognitive radio networks, capacity scaling law

\section{INTRODUCTION}

A fundamental problem that has generated much interest in the wireless network research community is the asymptotic capacity problem. Such problem is concerned with the capacity scaling laws of the underlying wireless network, i.e., how the individual node's capacity scales as the number of nodes increases. The study of such scaling laws is considered critical to the fundamental understanding of the underlying network. Since the pioneering work by Gupta and Kumar [5] on simple single-channel single-radio (SC-SR) networks, there has been active research on studying capacity scaling laws for other types of networks, e.g., for multi-channel networks [1], [2], [10], and for wireless networks with various physical layer technologies [3], [11], [14], [18], [22].

In this paper, we are interested in exploring capacity scaling laws for a cognitive radio ad hoc network (CRN). Cognitive radio (CR) [20] is a revolutionary radio technology that enjoys unparalleled advantages over traditional hardware-based radio. It is envisioned that CR will be the core radio technology for wireless networks in the future. However, due to the flexibility (and thus much larger design space) that comes with a CRN, none of the existing capacity scaling law results can be easily extended to a CRN. For instance, the available frequency bands at one node in a CRN (which may include any unused bands by primary users) may not be identical to those at the other nodes in the network, due to its geographical location and local spectrum availability. Such heterogeneity in frequency bands at each node poses a significant challenge to analyze capacity bounds.

\section{A. Main Contributions}

In this paper, we study asymptotic capacity for a CRN under both the protocol and the physical models [5]. We consider a random multi-hop CRN with $n$ nodes, with each node acting as a source node and transmitting data to a randomly chosen destination node. The per-node throughput $\lambda(n)$ is defined as the data rate that can be sent from each source to its destination. We aim to analyze the maximum asymptotic pernode throughput (or per-node capacity). Our main contributions are summarized as follows.

- Under the protocol model, we show that the lower and upper bounds for $\lambda(n)$ in a random CRN are $\Omega\left(\frac{C_{\alpha}}{\sqrt{n \ln n}}\right)$ and $O\left(\frac{C_{\zeta}}{\sqrt{n \ln n}}\right)$, respectively (denoted as $\left.\lambda(n) \in\left[\Omega\left(\frac{C_{\alpha}}{\sqrt{n \ln n}}\right), O\left(\frac{C_{\zeta}}{\sqrt{n \ln n}}\right)\right]\right)$, where $C_{\alpha}$ is the minimum link capacity over all links and $C_{\zeta}$ is the sum of capacity over all available bands in the CRN. Note that we include the constant terms $C_{\alpha}$ and $C_{\zeta}$ in the scaling results to show their potential impacts (similar to that in [5] which includes a constant term $W$ ). The analysis of capacity bounds is quite challenging, due to heterogeneous available bands on each link in a random CRN. The main novelty in our analysis is the creation of two auxiliary networks $\zeta$ and $\alpha$ that help transform the original heterogeneous bands to homogenous bands on each link for asymptotic analysis. The conception of auxiliary network $\zeta$ is intuitive and enables us to extend the results by Gupta and Kumar [5] to obtain an upper bound. However, creating auxiliary network $\alpha$ for lower bound analysis is not trivial. We show that a naive approach will yield zero for the lower bound, which is not useful (see Section IV-B1). Instead, we offer a non-conventional approach to construct auxiliary network $\alpha$, which enables us to obtain a meaningful lower bound. Note that even after proper construction of auxiliary network $\alpha$, GuptaKumar's approach does not admit straightforward solutions and cannot be applied directly to obtain the capacity lower bound for the original CRN. This is because that although we can apply Gupta-Kumar's approach to obtain 
a capacity lower bound for the auxiliary network $\alpha$, there does not appear to be an obvious connection between this capacity lower bound (for auxiliary network $\alpha$ ) and that for the original heterogenous $\mathrm{CRN}$. One cannot make a straightforward claim that the capacity lower bound for auxiliary network $\alpha$ is also a capacity lower bound for the original CRN without a rigorous proof, since a feasible solution to $\alpha$ may not be feasible to the original CRN. To obtain a meaningful capacity lower bound for the original CRN, we propose a two-step approach: (1) find a feasible solution for auxiliary network $\alpha$; and (2) transform this solution to a feasible solution to the original CRN.

- Under the physical model, we focus on the general case where each node is allowed to perform independent power control. We show that $\lambda(n)$ in a random CRN is $\lambda(n) \in\left[\Omega\left(\frac{C_{\alpha}}{\sqrt{n \ln n}}\right), O\left(\frac{C_{\zeta}}{n^{1 / \gamma}}\right)\right]$. In addition, we also give the result for the special case where power level is synchronized on all nodes in the network (as done in [5]). Our capacity analysis again hinges upon the two auxiliary networks $\zeta$ and $\alpha$. Again, the challenge here is the analysis for the lower bound. We exploit our earlier results for the protocol model and prove that by properly setting the interference range, a feasible solution to $\alpha$ under the protocol model is also a feasible solution under the physical model. Subsequently, we transform this feasible solution to $\alpha$ to a feasible solution to the original CRN and prove its correctness.

\section{B. Paper Organization}

The rest of this paper is organized as follows. Section II reviews related work on asymptotic capacity studies and offers a perspective of our work in relation to others. In Section III, we describe the details of network setting for a CRN in our study. In Section IV, we explore capacity scaling law under the protocol model. Built upon the results in Section IV, we further explore capacity scaling law under the physical model in Section V. Section VI concludes this paper.

\section{RELATED WORK}

Since the seminal work by Gupta and Kumar [5] on capacity scaling laws for a wireless network, there has been growing interest in this important area. In [5], two types of ad hoc networks, namely, random networks and arbitrary networks, are considered. In an arbitrary network [5], each node can be arbitrarily placed and can arbitrarily choose its destination. Thus, in order to maximize the network capacity, a node is always inclined to choose a nearby node as its destination to avoid multihop routing. Given the underlying assumption for an arbitrary network, its capacity has limited utility in practice. On the other hand, in a random network [5], the topology of the network is randomly generated and cannot be changed arbitrarily afterwards. Given its generality and more realistic assumption, its capacity results have application in practice. For this reason, we focus on random networks in this paper. Related work on random ad hoc networks can be further divided into the following two categories, namely, unicast capacity (e.g.,
[1], [2], [5], [10]) and multicast/broadcast capacity (e.g., [6], [12], [16], [23]). Given the focus of this paper is on unicast capacity, we will mainly review related work in this category.

In [5], Gupta and Kumar showed that for a random network, its capacity is $\Theta\left(\frac{C}{\sqrt{n \ln n}}\right)$ under the protocol model, and is $\left[\Omega\left(\frac{C}{\sqrt{n \ln n}}\right), O\left(\frac{C}{\sqrt{n}}\right)\right]$ under the physical model with synchronized power control, where $C$ is the channel capacity. In Sections IV and V, we will show that the result in [5] is a special case of ours.

In [1], [2], [10], unicast capacity of multi-channel ad hoc networks was investigated under the protocol model. In [1], Bhandari and Vaidya studied the capacity of multi-channel single-radio (MC-SR) networks where there are a set of channels in the network and each node can only switch to a subset of these channels. In [10], Kyasanur and Vaidya studied unicast capacity of multi-channel multi-radio (MCMR) networks where the number of bands used at a node is limited by the number of radios at the node. In [2], Dai et al. extended the work of [10] on MC-MR networks with consideration of directional antennas. All these prior efforts are based on traditional hardware-based radios. There are some fundamental differences between these prior efforts and ours in this paper. First, in these prior works, the set of channels available to each radio is the same as those on the other radios in the network, while in our CRN, the set of bands available at each node may be different. This difference is the result of traditional hardware-based radio's inability to perform spectrum sensing and thus must be pre-assigned to a set of channels on each node, while in a CRN, each node can perform spectrum sensing and may have a different set of available frequency bands depending on spectrum availability at its location (i.e., bands unused by primary users). Second, for traditional hardware-based radio, each radio interface has limited capability, e.g., one radio can only work on one channel at any time and may not be able to switch channel on a perpacket basis. That is, at any time instance, the number of bands that can be active at a node is limited by the number of radios at the node. On the other hand, a CR that we consider in this paper is assumed to be much more powerful and can work on many bands at the same time. That is, a CR can access to many more bands simultaneously than a hardware-based radio.

To date, work on asymptotic capacity for CRNs remains very limited. In [17], Vu et al. studied the capacity of a CRN for the simple single-hop case, where $n$ pairs of cognitive transmitter and receiver wish to communicate simultaneously in the presence of a single primary transmitter-receiver link. They showed that the sum-rate of the $n$ cognitive links scales linearly with $n$ as $n \rightarrow \infty$. In [21], Yin et al. considered a secondary $\mathrm{CRN}$ co-located with a primary ad hoc network under the assumption that the primary network must be sparser than the secondary network. They showed that the primary network and the secondary network both can achieve the same throughput scaling law as what Gupta and Kumar [5] established for a stand-alone wireless network. Jeon et al. [8] studied both the case where the primary network is an ad hoc network and the case where the the primary network is an 
infrastructure-supported wireless network. They also showed that the primary network and the secondary network both can simultaneously achieve the same throughput scaling law as a stand-alone ad hoc network regardless of whether the primary network is an ad hoc network or an infrastructuresupported network. However, the general case where there are heterogenous available bands is not considered. Further, the assumption that the secondary network is denser than the primary network may be overly restrictive.

Finally, there have been some recent efforts on studying capacity scaling laws under specialized wireless communication technologies, e.g., Gaussian channel model [3] (via adaptive modulation and coding scheme), ultra-wide band (UWB) [22], directional antennas [15], multiple-input multioutput (MIMO)[9], [14], network coding [11], and multiplepacket reception (MPR) [18]. These works are orthogonal to ours (on CR) and thus naturally lead to different results on capacity scaling laws.

\section{NETWORK MODEL}

We consider a CRN consisting of $n$ nodes positioned randomly in a unit square area. In a $\mathrm{CRN}$, each node senses a set of spectrum bands that it can use [13], which may include the set of bands not used by primary users. Thus, a CRN has a unique property that the set of available frequency bands $\mathcal{B}_{i}$ at a node $i$ depends on its location and may not be identical to that of another node in the network. A node $i$ can transmit to a node $j$ on a particular band only if this band is available to both nodes $i$ and $j$. More formally, denote $\mathcal{B}_{i j}=\mathcal{B}_{i} \cap \mathcal{B}_{j}$ the set of common available bands at both nodes $i$ and $j$. We have the following constraint.

A band $m$ can be used on link $(i, j)$ only if $m \in \mathcal{B}_{i j}$.

This constraint must be considered explicitly for a CRN, although it is implicitly assumed under wireless networks with traditional radios. Note that we only consider a static instance of a CRN. The temporal dynamics of available bands at each node over time is not considered and will be explored in future work.

Denote $\mathcal{E}$ the set of all links in a CRN, and $\mathcal{B}=\bigcup_{(i, j) \in \mathcal{E}} \mathcal{B}_{i j}$ the union of available bands over all links in the network. Note that $\mathcal{B}$ does not include those isolated bands that are available at a node but not at its neighbor(s). For a CRN, the bandwidth of each band may also be different, leading to different capacities on different bands. Denote $C_{m}$ the capacity on band $m$ and $C_{i j}=\sum_{m \in \mathcal{B}_{i j}} C_{m}$, i.e, the aggregate link capacity on link $(i, j)$ if it uses all its available bands.

For interference modeling, we consider both the protocol model and the physical model. Under the protocol model, a node $i$ can successfully transmit data to a node $j$ on a band $m \in \mathcal{B}_{i j}$ at time $t$ if and only if

- Receiving node $j$ is within the transmission range of node $i$, i.e.,

$$
d_{i j} \leq r(n)
$$

where $d_{i j}$ is the distance between nodes $i$ and $j$ and $r(n)$ is the transmission range. Constraint (2) implicitly sets a constraint on routing, i.e., it defines a set of possible candidate nodes (within the transmission range) as the next-hop node.

- For any other link $(k, l)$ that is active on band $m$ at the same time, it is required that $d_{k j} \geq(1+\Delta) r(n)$, where $(1+\Delta) r(n)$ represents an interference range, which is intended to keep concurrent transmitting node $k$ farther away from producing non-negligible interference on node $j$. More formally, we have

$$
\begin{aligned}
& d_{k j} \geq(1+\Delta) r(n) \text { for each link }(k, l) \in \mathcal{E}^{m}(t) \\
& \text { and }(k, l) \neq(i, j),
\end{aligned}
$$

where $\mathcal{E}^{m}(t)$ is the set of links in the network that are active on band $m$ at time $t$. (3) implicitly sets a constraint on scheduling.

Note that under the protocol model, the transmission range $r(n)$ is assumed to be the same at all nodes. Thus, the same (synchronized) transmission power $p(n)$ is used at all nodes.

Under the physical model, each node is allowed to perform independent power control. Denote $p_{i j}^{m}(t)$ the power used by node $i$ to transmit to node $j$ on band $m \in \mathcal{B}_{i j}$ at time $t$. Such a transmission is successful if and only if the signal-tointerference-and-noise-ratio (SINR) satisfies

$$
\operatorname{SINR}_{i j}=\frac{g_{i j} \cdot p_{i j}^{m}(t)}{\eta+\sum_{\substack{k, l) \neq(i, j) \\(k, l) \in \mathcal{E}^{m}(t)}}^{(k)} g_{k j} \cdot p_{k l}^{m}(t)} \geq \beta,
$$

where $g_{i j}=d_{i j}^{-\gamma}$ is the channel gain over link $(i, j), \gamma \geq 2$ is the path loss index, $\eta$ is the ambient noise power, and $\beta$ is the SINR threshold.

Under the above setting for a CRN, we consider a common throughput $\lambda(n)$ for each node (source) in the network to its randomly selected destination. The goal of capacity scaling law is to find the maximum $\lambda(n)$ that can be achieved by considering multi-hop routing and time-slotted scheduling.

\section{ASYMPTOTIC CAPACITY UNDER THE PROTOCOL MODEL}

In this section, we analyze the capacity scaling law under the protocol model. As discussed in Section I, the root of the difficulties associated with a CRN is the heterogeneity of available bands among the links. To overcome this difficulty, we create two auxiliary networks $\zeta$ and $\alpha$ to facilitate our analysis. In Section IV-A, we develop a capacity upper bound with the help of auxiliary network $\zeta$. The lower bound analysis is given in Section IV-B, which is more challenging and interesting. The main difficulty here is how to create an appropriate auxiliary network $\alpha$ to facilitate the analysis and how to use this auxiliary network $\alpha$ to obtain a capacity lower bound.

The result of this section can be summarized as follows (see Theorems 1 and 2).

Under the protocol model, the capacity of a CRN with $n$ nodes is $\lambda(n) \in\left[\Omega\left(\frac{C_{\alpha}}{\sqrt{n \ln n}}\right), O\left(\frac{C_{\zeta}}{\sqrt{n \ln n}}\right)\right]$ almost surely when $n \rightarrow \infty$. 


\section{A. A Capacity Upper Bound}

To address the heterogeneity problem in each node's available bands, we propose to construct an auxiliary network to facilitate our analysis. Our goal is to have an auxiliary network with the following two characteristics: (i) homogeneous bands setting, i.e., the sets of available bands on all links are the same; (ii) given a CRN, its capacity is upper bounded by its corresponding auxiliary network. If we can get such an auxiliary network, then we can focus on developing the capacity upper bound for this auxiliary network with homogenous bands setting, which is also a capacity upper bound for the original CRN.

Such an auxiliary network can be constructed by adding some extra bands to the set of available bands on each link so that the sets of available bands on all the links are identical. More formally, we have the following definition for this auxiliary network.

Definition 1: (Auxiliary network $\zeta$ ) An auxiliary network $\zeta$ contains all the nodes and links in the original CRN and the same source-destination pairs. The set of available bands on all links in auxiliary network $\zeta$ is defined as $\mathcal{B}_{\zeta}$, where $\mathcal{B}_{\zeta}=\bigcup_{(i, j) \in \mathcal{E}} B_{i j}$, i.e., the set of all available bands in the network.

Since the frequency band resource in this auxiliary network is no less than the original CRN, we have the following lemma.

Lemma 1: Under the protocol model, for any given CRN (with heterogenous available bands on each link), its capacity is no more than the capacity of its corresponding auxiliary network $\zeta$.

Lemma 1 is easily verified since any feasible routing and scheduling solution to a CRN is also feasible to its corresponding auxiliary network $\zeta$. Given the homogenous auxiliary network $\zeta$, we can now apply the result by Gupta and Kumar [5] and obtain its capacity upper bound as $O\left(\frac{C_{\zeta}}{\sqrt{n \ln n}}\right)$, where $C_{\zeta}=\sum_{m \in \mathcal{B}_{\zeta}} C_{m}$. Based on Lemma 1 and this capacity upper bound for $\zeta$, we have the following theorem.

Theorem 1: Under the protocol model, a capacity upper bound for a CRN with $n$ nodes is $O\left(\frac{C_{\zeta}}{\sqrt{n \ln n}}\right)$ almost surely when $n \rightarrow \infty$.

\section{B. A Constructive Lower Bound}

The solution to find a lower bound is much more difficult. Note that, to develop a capacity lower bound for a CRN under the protocol model, it is sufficient to construct a feasible solution that satisfies all the constraints (see Section III). This feasible solution can thus serve as a lower bound. But due to the heterogeneity of available bands on different links, band assignment becomes a very difficult problem if we want to construct a feasible solution directly.

1) Construct Auxiliary Network $\alpha$ : Recognizing that a direct approach to construct a feasible solution is not likely to be fruitful, we again resort to creating a homogeneous auxiliary network to facilitate our analysis. However, such a homogeneous auxiliary network is not so straightforward this time, as we illustrate in the following naive approach.

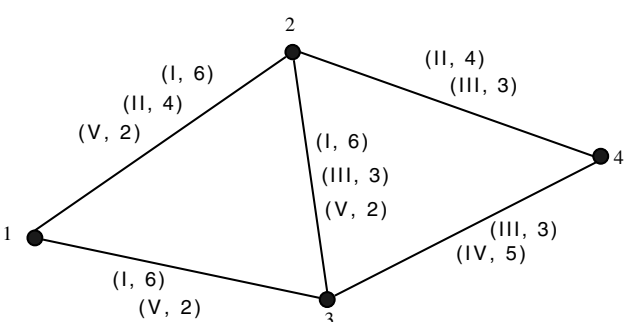

(a) A 4-node CRN, where $\mathcal{B}_{1}=\{\mathrm{I}, \mathrm{II}, \mathrm{V}\}, \mathcal{B}_{2}=$ $\{$ I, II, III, V $\}, \mathcal{B}_{3}=\{$ I, III, IV, V $\}$ and $\mathcal{B}_{4}=$ $\{$ II, III, IV $\}$.

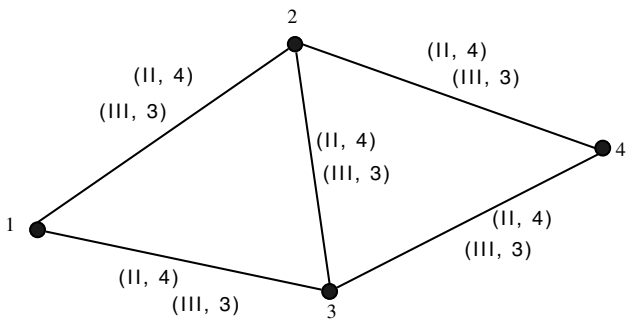

(b) The corresponding auxiliary network $\alpha$, where the set of available bands at each node is $\{$ II, III $\}$.

Fig. 1. An example illustrating how to construct auxiliary network $\alpha$.

A naive approach: Recall that in the development of a capacity upper bound, we define the available bands on each link in the auxiliary network $\zeta$ to be the union of available bands on all the links in the network. To get a capacity lower bound, one might consider to define the available bands on each link in the auxiliary network $\alpha$ by taking the intersection of available bands among all the links in the network. Unfortunately, such a approach may not work as it may lead to an empty set. In this case, we will have a trivial 0 capacity as lower bound, which is not useful. As an example, consider the simple 4-node CRN in Fig. 1(a). The available bands for each link are the common bands at two nodes. We show the available bands on each link and corresponding capacity of each band in Fig. 1(a). For instance, the bands on link $(1,2)$ is the intersection of available bands on nodes 1 and 2, which are I, II, and V. The capacity of band I is 6 , the capacity of band II is 4 , and the capacity of band $\mathrm{V}$ is 2 , respectively. We can see that although there are some bands on each link, there does not exist a single band that is common on all links.

Our approach: In our approach, we first identify the bottleneck link with the minimum capacity in the original CRN. We then use the set of available bands on the bottleneck link to construct an auxiliary network. We again use the CRN in Fig. 1(a) as an example. The bottleneck link in the network is the link between nodes 2 and 4, which has the smallest capacity (i.e., $4+3=7$ ) among all the links in the network. To construct the auxiliary network, we retain the same topology structure, except that the set of available bands on each link is now set to the same as that on the bottleneck link in the original CRN (see Fig. 1(b)). Although such construction may appear strange at first glance, its benefits will soon become clear when we analyze the lower bound (see Lemma 5). 
More formally, we define our auxiliary network as follows.

Definition 2: (Auxiliary network $\alpha$ ) An auxiliary network $\alpha$ contains all the nodes and links in the original CRN and the same source-destination pairs. The set of available bands on each link in the auxiliary network is defined as $\mathcal{B}_{\alpha}=\mathcal{B}_{i j}$, where link $(i, j)$ denotes the bottleneck link in the original $C R N$, i.e., $C_{i j}=\min \left\{C_{k l}, \forall(k, l) \in \mathcal{E}\right\}$.

Comparing link capacities in the original CRN and in the auxiliary network, we have the following property.

Property 1: For the capacity of each link $(k, l)$ in the original CRN, we have $C_{k l} \geq C_{\alpha}$, where $C_{\alpha}$ is defined as $\sum_{m \in \mathcal{B}_{\alpha}} C_{m}$.

Note that we do not consider the trivial case where a node does not have any band in common with its neighboring nodes. Such isolated node will lead to a zero capacity.

For the homogenous auxiliary network $\alpha$, we can again apply the result in [5] to obtain its capacity lower bound. A capacity lower bound (for auxiliary network $\alpha$ ) does not offer us a straightforward solution to a capacity lower bound to the original CRN, which is our main interest. Further, there does not appear to be an obvious connection between the two since a feasible solution to the auxiliary network $\alpha$ is, in general, not feasible to the original CRN.

To derive a capacity lower bound for the original CRN, we propose a two-step approach: (1) find a feasible solution for auxiliary network $\alpha$; and (2) transform this solution to a feasible solution to the original CRN. We now elaborate each step in the rest of this section.

2) Find a Feasible Solution for Auxiliary Network $\alpha$ : We now construct a feasible solution $\psi_{\alpha}$ for auxiliary network $\alpha$. In this solution, we need to design both a routing scheme and a scheduling scheme. Our routing scheme follows a cellbased approach. That is, we divide the unit square area into small cells such that there is at least one node in each cell almost surely when $n \rightarrow \infty$ (see Lemma 3 ). For each sourcedestination pair, the straight line that connects them will pass through a number of cells. One node is chosen from each of these cells as a relay for multi-hop routing.

To avoid interference, scheduling must be performed along with cell-based routing. We can build a conflict graph to model interference relationships among the links, with each vertex representing a link in the auxiliary network and each edge between two vertices representing a conflict. We analyze the maximum number of conflict links to a link (or the maximum degree in the conflict graph) and then apply the result for graph coloring to obtain a scheduling scheme. Afterward, we obtain a complete solution with the following throughput.

Lemma 2: Under the protocol model, we can construct a feasible solution $\psi_{\alpha}$ to auxiliary network $\alpha$ with a throughput of $\lambda_{\alpha}(n)=\Omega\left(\frac{C_{\alpha}}{\sqrt{n \ln n}}\right)$.

Now we give details for routing and scheduling in the above discussion.

Routing Scheme: We develop a cell-based routing scheme. We divide the unit square into small cells of square size $a(n)=\sqrt{\frac{n}{\ln n}} \times \sqrt{\frac{n}{\ln n}}=\frac{\ln n}{n}$ and set the transmission range

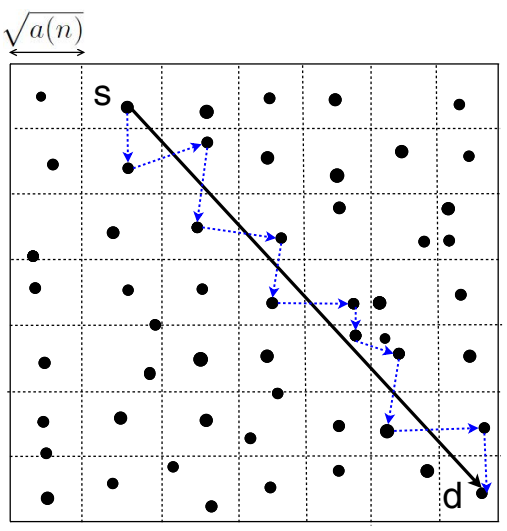

Fig. 2. Multi-hop routing from a source node $s$ to its destination node $d$.

$r(n)=\sqrt{5 a(n)}$ so that any node in one cell can transmit to another node in its four neighboring cells. Now, we draw a line to connect each source-destination pair, which passes through some cells. One node is chosen from each of these cells to relay the traffic from the source node to its destination. See Fig. 2 as an example.

Such a routing scheme requires at least one node in each cell. We call a cell without any node an "empty" cell. We have the following lemma.The proof is similar to the proof of Claim 3.1 in [7] and is omitted here.

Lemma 3: For auxiliary network $\alpha$ with $n$ nodes and the cell size of $a(n)=\frac{\ln n}{n}$, there is no empty cell almost surely when $n \rightarrow \infty$. That is, the routing scheme is feasible almost surely when $n \rightarrow \infty$.

Scheduling Scheme: In our solution, we design the same scheduling scheme for all bands, i.e., once a link is active, it treats all bands in $\mathcal{B}_{\alpha}$ as one aggregate band. We consider time-slot based scheduling, i.e., we divide one time frame into multiple time slots to satisfy the protocol model scheduling constraint (3).

To analyze the performance of our scheduling scheme, we need to know the number of conflicting links for each link $(i, j)$, which directly affects the number of time slots for scheduling and capacity. Two links are in conflict if they cannot be active on the same band at the same time. Then, we have the following lemma on the number of conflicting links for any link in the network. This lemma can be proved directly by combining Lemmas 2 and 3 in [4], and is omitted here due to limited space.

Lemma 4: Under our routing scheme for auxiliary network $\alpha$ with $n$ nodes, the number of conflicting links for any link is upper bounded by $O(n \sqrt{a(n)})$ almost surely when $n \rightarrow \infty$.

To schedule these conflict links, we use a conflict graph to model them. Each link in the auxiliary network corresponds to a vertex in the conflict graph, and any conflict in the auxiliary network is represented by an edge connecting two corresponding vertices in the conflict graph. If we use different vertex color to represent each time slot, then the scheduling problem reduces to the well-studied vertex-color problem. Using Lemma 4, the degree of each vertex in the conflict graph 
will be at most $c_{2} \cdot n \sqrt{a(n)}$ for some constant $c_{2}$ almost surely when $n \rightarrow \infty$. Then the required number of colors is at most $1+c_{2} \cdot n \sqrt{a(n)}$ [19]. So we can divide one time frame into at most $1+c_{2} \cdot n \sqrt{a(n)}$ equal length time slots for scheduling. Therefore, the achievable throughput $\lambda_{\alpha}(n)$ is given by

$$
\begin{aligned}
\lambda_{\alpha}(n) & \geq \frac{C_{\alpha}}{1+c_{2} \cdot n \sqrt{a(n)}}=\frac{C_{\alpha}}{1+c_{2} \sqrt{n \ln n}} \\
& =\Omega\left(\frac{C_{\alpha}}{\sqrt{n \ln n}}\right),
\end{aligned}
$$

where the second equality holds because $a(n)=\frac{\ln n}{n}$.

3) Obtain a Feasible Solution to the Original CRN: In the last step, we find a feasible solution $\psi_{\alpha}$ to the auxiliary network that has a throughput of $\lambda_{\alpha}(n)=\Omega\left(\frac{C_{\alpha}}{\sqrt{n \ln n}}\right)$. Based on $\psi_{\alpha}$, we now define a feasible solution $\psi$ to the original CRN. In $\psi$, for routing, we follow the same routing scheme as that in $\psi_{\alpha}$. For scheduling, if a link $(i, j)$ is active on all bands in $\mathcal{B}_{\alpha}$ in a time slot under solution $\psi_{\alpha}$ (recall that in $\psi_{\alpha}$, once a link is active, it uses all bands in $\mathcal{B}_{\alpha}$ ), then we let this link be active on all bands in $\mathcal{B}_{i j}$ in the same time slot under solution $\psi$. Given that each link has more diverse bands in the original CRN than in $\alpha$, solution $\psi$ constructed in this manner is also feasible. This result is stated in the following lemma.

Lemma 5: The constructed $\psi$ is a feasible solution to the original CRN under the protocol model and its throughput $\lambda(n)=\lambda_{\alpha}(n)$.

Proof: To show $\psi$ is feasible, we need to show that constraints (1), (2), and (3) hold under $\psi$. Based on the construction of $\psi$, it is clear that band assignment constraint (1) holds. Furthermore, routing constraints (2) also holds under $\psi$ by the construction of $\psi$ and the feasibility of $\psi_{\alpha}$. Before we show that scheduling constraint (3) holds under $\psi$, we need to analyze the relationship between $\mathcal{E}_{\psi}^{m}(t)$ (the set of active links on a band $m$ at time $t$ under $\psi$ ) and $\mathcal{E}_{\psi_{\alpha}}(t)$ (the set of active links at time $t$ under $\psi_{\alpha}$ ). Note that $\mathcal{E}_{\psi}^{m}(t)$ is different for different band $m$. But under $\psi_{\alpha}$, once a link is active, it uses all bands in $\mathcal{B}_{\alpha}$. Thus, $\mathcal{E}_{\psi_{\alpha}}(t)$ does not depend on bands. Based on the construction of $\psi$, we have that a link $(i, j) \in \mathcal{E}_{\psi}^{m}(t)$ if and only if $m \in \mathcal{B}_{i j}$ and under $\psi_{\alpha}$, link $(i, j)$ is active at time $t$ under $\mathcal{B}_{\alpha}$. Thus, we have

$$
\mathcal{E}_{\psi}^{m}(t)=\left\{(i, j): m \in \mathcal{B}_{i j},(i, j) \in \mathcal{E}_{\psi_{\alpha}}(t)\right\} \subseteq \mathcal{E}_{\psi_{\alpha}}(t) .
$$

Now we are able to show that (3) also holds under $\psi$. Consider a link $(i, j) \in \mathcal{E}_{\psi}^{m}(t)$. It is clear from (5) that any other link $(k, l) \in \mathcal{E}_{\psi}^{m}(t)$ is also in $\mathcal{E}_{\psi_{\alpha}}(t)$, i.e., a link that is active on band $m$ at time $t$ under $\psi$ is also active at time $t$ under $\psi_{\alpha}$. Then, since (3) holds under $\psi_{\alpha}$, (3) should also hold under $\psi$. Thus, solution $\psi$ is feasible.

Finally, we analyze the throughput achieved by $\psi$. For each link $(i, j)$, since we have $C_{i j} \geq C_{\alpha}$ by Property 1 , the achieved capacity on each link $(i, j)$ under $\psi$ is no less than that under $\psi_{\alpha}$. Since link capacity constraint holds on each link under $\psi_{\alpha}$ and the same routing scheme is used in $\psi$, link capacity constraint also holds on each link under $\psi$. Thus, the same throughput $\lambda(n)=\lambda_{\alpha}(n)$ is achieved by $\psi$.
Combining Lemmas 2 and 5, we have the following capacity lower bound for a CRN.

Theorem 2: Under the protocol model, the capacity of a $C R N$ is $\lambda(n)=\Omega\left(\frac{C_{\alpha}}{\sqrt{n \ln n}}\right)$ almost surely when $n \rightarrow \infty$.

\section{Asymptotic Capacity under the Physical Model}

In this section, we analyze the capacity scaling law under the physical model. We consider both the special case where nodes are allowed to perform synchronized power control and the general case where each node is allowed to perform independent power control. For the spacial case, the result can be summarized as follows (also see Theorems 3 and 5).

Under the physical model with synchronized power control at all nodes, the capacity of a CRN with $n$ nodes is $\lambda(n) \in$ $\left[\Omega\left(\frac{C_{\alpha}}{\sqrt{n \ln n}}\right), O\left(\frac{C_{\zeta}}{\sqrt{n}}\right)\right]$ almost surely when $n \rightarrow \infty$.

For the general case, the result can be summarized as follows (also see Theorems 4 and 5).

Under the physical model with independent power control at each node, the capacity of a CRN with $n$ nodes is $\lambda(n) \in$ $\left[\Omega\left(\frac{C_{\alpha}}{\sqrt{n \ln n}}\right), O\left(\frac{C_{\zeta}}{n^{1 / \gamma}}\right)\right]$ almost surely when $n \rightarrow \infty$, where $\gamma$ is the path loss index.

\section{A. Finding An Upper Bound}

We again employ auxiliary network $\zeta$ (see Definition 1) to facilitate our analysis. Similar to Lemma 1 for the protocol model, we have the following lemma for the physical model.

Lemma 6: Under the physical model, for any given CRN, its capacity is no more than the capacity of its corresponding auxiliary network $\zeta$, regardless of whether independent or synchronized power control at each node.

For the homogenous auxiliary network $\zeta$ with synchronized power control, we can apply the results in [5] and obtain its capacity upper bound as $O\left(\frac{C_{\zeta}}{\sqrt{n}}\right)$. Then based on Lemma 6 , we have the following capacity upper bound for the original CRN.

Theorem 3: Under the physical model with synchronized power control at all nodes, a capacity upper bound for a CRN with $n$ nodes is $O\left(\frac{C_{\zeta}}{\sqrt{n}}\right)$ almost surely when $n \rightarrow \infty$.

For the general case with independent power control on each node, we have the following lemma for auxiliary network $\zeta$. The proof of this lemma is similar to the proof of Theorem 2.1 in [5], and is omitted here due to limited space.

Lemma 7: Under the physical model with independent power control at each node, the capacity of auxiliary network $\zeta$ is $\lambda_{\zeta}(n)=O\left(\frac{C_{\zeta}}{n^{1 / \gamma}}\right)$ almost surely when $n \rightarrow \infty$.

Combining Lemmas 6 and 7, we have the following theorem.

Theorem 4: Under the physical model with independent power control on each node, the capacity of a CRN is $\lambda(n)=$ $O\left(\frac{C_{\zeta}}{n^{1 / \gamma}}\right)$ almost surely when $n \rightarrow \infty$.

\section{B. Construction of A Lower Bound}

Similar to the case under the protocol model, we employ our auxiliary network $\alpha$ (see Definition 2) and follow the twostep approach (as we did in Section IV-B) to construct a lower bound, although the details are different. 


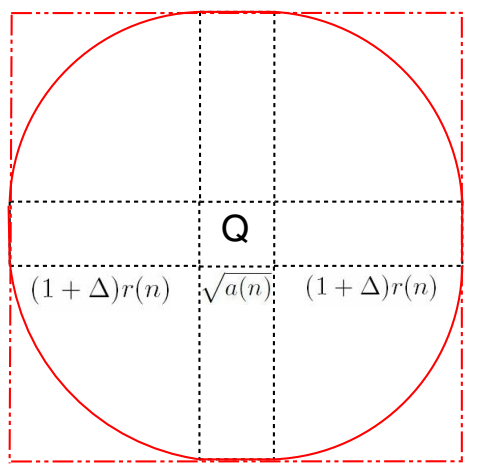

Fig. 3. The area to cover all the transmitting nodes that may interfere with the receivers in cell $Q$.

Step 1: A Feasible Solution for Auxiliary Network $\alpha$. We now need to construct a feasible solution for auxiliary network $\alpha$. However, constructing a feasible solution for the physical model is more difficult than that for the protocol model. This is because that under the protocol model, we only need to consider distance in scheduling constraint (3). But under the physical model, we also need to consider power control in the SINR constraint (4).

Given that we have developed a feasible solution $\psi_{\alpha}$ for the protocol model, we hope to use this result and develop a feasible solution for the physical model. We observe that if we set the parameter $\Delta$ in the protocol model "large enough", then $\psi_{\alpha}$ is also a feasible solution for the physical model. This is because a large $\Delta$ will impose more constraints on conflict graph, and thus reduce interference from neighboring nodes in the protocol model solution. As a result, the SINR at a receiver can be made large enough to satisfy the physical model SINR constraint (4). In this case, $\psi_{\alpha}$ is also a feasible solution for the physical model. This insight is the basis of the following lemma.

Lemma 8: Under the physical model, we can construct a feasible solution $\psi_{\alpha}$ to auxiliary network $\alpha$ with a capacity of $\lambda_{\alpha}(n)=\Omega\left(\frac{C_{\alpha}}{\sqrt{n \ln n}}\right)$.

Proof: In this proof, we show that if we set

$$
\Delta \geq\left\{\frac{p(n) \beta}{2 r(n)^{2}\left[p(n)-\beta \eta r(n)^{\gamma}\right]}\right\}^{\frac{1}{2+\gamma}}-1,
$$

where $p(n)$ is the transmission power used in the protocol model solution $\psi_{\alpha}$, then the solution $\psi_{\alpha}$ developed for the protocol model (in Section IV-B2) is also feasible under the physical model.

First, we show that the physical model SINR constraint (4) is satisfied under $\psi_{\alpha}$. Based on the construction of $\psi_{\alpha}$ in Section IV-B2, we know that once a link $(i, j)$ is active, other nodes within a square with side length $2(1+\Delta) r(n)+\sqrt{a(n)}$, which is centered on the cell where node $j$ is located, cannot transmit (see Fig. 3). Note that node $j$ may be located on the boundary of the unit square. Thus, the number of links that make interference to link $(i, j)$ is at most

$$
\begin{aligned}
& {\left[\frac{2(1+\Delta) r(n)+1}{2(1+\Delta) r(n)+\sqrt{a(n)}}\right]^{2}-1 } \\
\leq & {\left[\frac{2(1+\Delta) r(n)+1}{2(1+\Delta) r(n)+\sqrt{a(n)}}\right]^{2}-1 } \\
= & \frac{[1-\sqrt{a(n)}][4(1+\Delta) r(n)+\sqrt{a(n)}+1]}{[2(1+\Delta) r(n)+\sqrt{a(n)}]^{2}} \\
< & \frac{2}{[2(1+\Delta) r(n)+\sqrt{a(n)}]^{2}} \\
< & \frac{2}{4[(1+\Delta) r(n)]^{2}}=\frac{1}{2[(1+\Delta) r(n)]^{2}},
\end{aligned}
$$

where the third inequality holds by $1-\sqrt{a(n)}<1$ and $4(1+$ $\Delta) r(n)+\sqrt{a(n)} \rightarrow 0<1$ when $n \rightarrow \infty$. Based on the construction of $\psi_{\alpha}$, the interference at receiving node $j$ from each of these links will be at most $\frac{p(n)}{[(1+\Delta) r(n)]^{\gamma}}$. Therefore, we have

$$
\begin{aligned}
\operatorname{SINR}_{i j} & >\frac{\frac{p(n)}{[r(n)]^{\gamma}}}{\eta+\frac{1}{2[(1+\Delta) r(n)]^{2}} \frac{p(n)}{[(1+\Delta) r(n)]^{\gamma}}} \\
& =\frac{r(n)^{-\gamma}}{\eta p(n)^{-1}+\frac{1}{2}[(1+\Delta) r(n)]^{-(2+\gamma)}} \\
& \geq \frac{r(n)^{-\gamma}}{\eta p(n)^{-1}+\left[\beta^{-1} r(n)^{-\gamma}-\eta p(n)^{-1}\right]}=\beta,
\end{aligned}
$$

where the third inequality holds by (6). Thus, under $\psi_{\alpha}$, for each link $(i, j)$ that is active on a band $m \in \mathcal{B}_{i j}$ at time $t$, the SINR constraint (4) holds. Therefore, the power control and scheduling schemes in $\psi_{\alpha}$ are also feasible under the physical model. Furthermore, the achieved capacity on each link under the physical model is equal to that under the protocol model. As a result, link capacity constraint is still satisfied on each link under the physical model and the achieved throughput under the physical model will be the same as that under the protocol model, which is $\Omega\left(\frac{C_{\alpha}}{\sqrt{n \ln n}}\right)$ almost surely when $n \rightarrow \infty$.

In this solution, the same transmission power $p(n)$ is used at all nodes (i.e., synchronized power control). Note that a feasible solution under synchronized power control is also a feasible solution under independent power control. Thus, under the physical model, a lower bound on the capacity of auxiliary network $\alpha$ is $\lambda_{\alpha}(n)=\Omega\left(\frac{C_{\alpha}}{\sqrt{n \ln n}}\right)$ almost surely when $n \rightarrow \infty$ for both independent power control and synchronized power control.

Step 2: Obtain a Feasible Solution to the Original CRN. We now need to transform the feasible solution constructed in Step 1 for auxiliary network $\alpha$ to a feasible solution to the original CRN. This transformation is similar to that in Section IV-B3, except that we need to verify that the constructed solution is feasible for the physical model, i.e., the SINR constraint (4). We state our result in the following lemma. 
Lemma 9: Under the physical model, we can construct a feasible solution $\psi$ to the original CRN with a throughput $\lambda(n)=\lambda_{\alpha}(n)$.

Proof: We construct $\psi$ by using the same routing scheme as that in $\psi_{\alpha}$. The power control and scheduling schemes in $\psi$ are constructed as follows. Let each link $(i, j)$ be active on all bands in $\mathcal{B}_{i j}$ at time $t$ whenever it is active on all bands in $\mathcal{B}_{\alpha}$ under $\psi_{\alpha}$. Also, we set the transmission power $p(n)$ as that in $\psi_{\alpha}$.

For a link $(i, j)$ that is active on a band $m$ at time $t$ under $\psi$, its SINR is

$$
\begin{aligned}
\operatorname{SINR}_{i j}(\psi) & =\frac{g_{i j} \cdot p(n)}{\eta+\sum_{(k, l) \in \mathcal{E}_{\psi}^{m}(t)}^{(k, l) \neq(i, j)} g_{k j} \cdot p(n)} \\
& \geq \frac{g_{i j} \cdot p(n)}{\eta+\sum_{(k, l) \in \mathcal{E}_{\psi_{\alpha}}(t)}^{(k, l) \neq(i, j)} g_{k j} \cdot p(n)} \\
& =\operatorname{SINR}_{i j}\left(\psi_{\alpha}\right) \geq \beta,
\end{aligned}
$$

where the second inequality holds because $\mathcal{E}_{\psi}^{m}(t) \subseteq \mathcal{E}_{\psi_{\alpha}}(t)$ in (5), and the last inequality holds because $\psi_{\alpha}$ is feasible. Thus, under $\psi$, for each link $(i, j)$ that is active on a band $m \in \mathcal{B}_{i j}$ at time $t$, the SINR constraint (4) holds. Therefore, the power control and scheduling schemes in $\psi$ are feasible.

For each link $(i, j)$, since we have $C_{i j} \geq C_{\alpha}$ in Property 1 , the total achieved link capacity under $\psi$ is no less than that under $\psi_{\alpha}$. Since the same routing scheme is used, this routing scheme in $\psi$ is also feasible. Thus, $\psi$ is a feasible solution with a throughput $\lambda_{\alpha}(n)$. Again, since the same transmission power $p(n)$ is used at all nodes, this solution is a feasible solution under both synchronized power control and independent power control. Thus, for the physical model, a lower bound for the capacity of a CRN is $\lambda(n)=\lambda_{\alpha}(n)$ under both independent power control and synchronized power control.

Based on Lemmas 8 and 9, we have the following theorem on lower bound of a CRN capacity.

Theorem 5: Under the physical model, the capacity of a $C R N$ is $\lambda(n)=\Omega\left(\frac{C_{\alpha}}{\sqrt{n \ln n}}\right)$ almost surely when $n \rightarrow \infty$.

\section{CONCLUSION}

In this paper, we studied capacity scaling laws for cognitive radio ad hoc networks (CRNs) under both the protocol and the physical models, respectively. The main novelties in this work are: (1) the design of suitable auxiliary networks to analyze a network with heterogeneous bands, and (2) the analysis of relationship between solutions under the auxiliary networks and solutions to the original CRNs. Although our results for CRN share similar form to those results by Gupta-Kumar for single-channel single-radio network, our problem is much more challenging and the approach is far beyond a simple extension of that in [5]. The results in this paper fill an important gap in the fundamental understanding of CRNs.

\section{ACKNOWLEDGMENTS}

The work of Y.T. Hou, Y. Shi, and C. Jiang was supported in part by Naval Research Lab (NRL) under Grant N0017310-1-G-007 and NSF under Grant CNS-0910531. The work of S. Kompella was supported in part by ONR.

\section{REFERENCES}

[1] V. Bhandari and N.H. Vaidya, "Capacity of multi-channel wireless networks with random $(c, f)$ assignment," in Proc. ACM MobiHoc, pp. 229-238, Montreal, Quebec, Canada, Sep. 9-14, 2007.

[2] H. Dai, K. Ng, R.C. Wong, and M. Wu, "On the capacity of multi-channel wireless networks using directional antennas" in Proc. IEEE INFOCOM, pp. 1301-1309, Phoenix, AZ, Apr. 13-18, 2008.

[3] M. Franceschetti, O. Dousse, and D.N.C. Tse, "Closing the gap in the capacity of wireless networks via percolation theory," IEEE Transaction on Information Theory, vol. 53, no. 3, pp. 1009-1018, March 2007.

[4] A.E. Gamal, J. Mammen, B. Prabhakar, and D. Shah, "Throughput-delay trade-off in wireless networks," in Proc. IEEE INFOCOM, pp. 464-475, Hong Kong, China, March 7-11, 2004.

[5] P. Gupta and P. Kumar, "The capacity of wireless networks," IEEE Transaction on Information Theory, vol. 46, no. 2, pp. 388-404, March 2000.

[6] A. Keshavarz-Haddad, V. Ribeiro, and R. Riedi, "Broadcast capacity in multihop wireless networks," in Proc. ACM MobiCom, pp. 239-250, Los Angeles, CA, Sep. 23-26, 2006.

[7] S.R. Kulkarni and P. Viswanath, "A deterministic approach to throughput scaling in wireless networks," IEEE Transaction on Information Theory, vol. 50, no. 6, pp. 1041-1049, June 2004.

[8] S.-W. Jeon, N. Devroye, M. Vu, S.-Y. Chung, and V. Tarokh, "Cognitive networks achieve throughput scaling of a homogeneous network," 5 pages, 7th Intl. Symposium on Modeling and Optimization in Mobile, Ad Hoc, and Wireless Networks (WiOpt), Seoul, Korea, June 2009.

[9] C. Jiang, Y. Shi, Y.T. Hou, and S. Kompella, "On the asymptotic capacity of multi-hop MIMO ad hoc networks," IEEE Transactions on Wireless Communications, vol. 10, no. 4, pp. 1032-1037, Apr. 2011.

[10] P. Kyasanur and N.H. Vaidya, "Capacity of multi-channel wireless networks: impact of number of channels and interfaces," in Proc. ACM MobiCom, pp. 43-57, Cologne, Germany, Aug. 28-Sep. 2, 2005.

[11] J. Liu, D. Goeckel, and D. Towsley, "Bounds on the gain of network coding and broadcasting in wireless networks," in Proc. IEEE INFOCOM, pp. 724-732, Anchorage, AK, May 6-12, 2007.

[12] X. Mao, X. Li, and S. Tang, "Multicast capacity for hybrid wireless networks," in Proc. ACM MobiHoc, pp.189-198, Hong Kong, China, May 26-30, 2008.

[13] S.M. Mishra, A. Sahai, and R.W. Brodersen, "Cooperative sensing among cognitive radios," in Proc. IEEE International Conference on Communications, pp. 1658-1663, Istanbul, Turkey, June 11-15, 2006.

[14] A. Özgür, O. Lévêque and D.N.C. Tse, "Hierarchical cooperation achieves optimal capacity scaling in ad hoc networks," IEEE Transaction on Information Theory, vol. 53, no. 10, pp. 3549-3572, Oct. 2007.

[15] C. Peraki and S.D. Servetto, "On the maximum stable throughput problem in random networks with directional antennas," in Proc. ACM MobiHoc, pp. 76-87, Annapolis, Maryland, June 1-3, 2003.

[16] S. Shakkottai, X. Liu, and R. Srikant, "The multicast capacity of large multihop wireless networks," in Proc. ACM MobiHoc, pp. 247-255, Montreal, Quebec, Canada, Sep. 9-14, 2007.

[17] M. Vu, N. Devroye, M. Sharif, and V. Tarokh, "Scaling laws of cognitive networks," International Conference on Cognitive Radio Oriented Wireless Networks and Communications (Crowncom), pp. 2-8, Orlando, FL, July 31-Aug. 3, 2007.

[18] Z. Wang, H.R. Sajadpour, and J.J. Garcia-Luna-Aceves, "The capacity and energy efficiency of wireless ad hoc networks with multi-packet reception," in Proc. ACM MobiHoc, pp. 179-188, Hong Kong, China, May 26-30, 2008.

[19] D.B. West, Introduction to Graph Theory, Prentice Hall, Upper Saddle River, NJ, 2001.

[20] A.M. Wyglinski, M. Nekovee, and Y.T. Hou, Cognitive Radio Communications and Networks: Principles and Practices, Academic Press/Elsevier, 2010. ISBN: 978-0-12-374715-0.

[21] C. Yin, L. Gao, and S. Cui, "Scaling laws of overlaid wireless networks: a cognitive radio network vs. a primary network", in Proc. IEEE GLOBECOM, pp. 1235-1239, New Orleans, LA, Nov. 30-Dec. 4, 2008.

[22] H. Zhang and J.C. Hou, "Capacity of wireless ad-hoc networks under ultra wide band with power constraint," in Proc. IEEE INFOCOM, pp. 455-465, Miami, FL, March 13-17, 2005.

[23] R. Zheng, "Information dissemination in power-constrained wireless network," in Proc. IEEE INFOCOM, Barcelona, Catalunya, Spain, April 2329, 2006. 